Case Study

\title{
Effects of lengthwise postural taping for lumbar spine rotation with flexion syndrome: a case report
}

\author{
Sun-Shil Shin ${ }^{1)}$, Won-Gyu Yoo ${ }^{1)^{*}}$ \\ 1) Department of Physical Therapy, College of Biomedical Science and Engineering, Inje University: \\ 197 Inje-ro, Gimhae-si, Gyeongsangnam-do 50834, Republic of Korea
}

\begin{abstract}
Purpose] This study investigated the effects of lengthwise postural taping on a patient having lumbar spine rotation with flexion syndrome during prolonged periods of sitting. [Subject and Methods] The subject was a 22-year-old woman who had developed transient low back pain in the lumbo-pelvic region. The subject was asked to sit in a chair during 30 minutes of computer work under three conditions: no taping, extensive lumbo-pelvic region taping, and lengthwise lumbo-pelvic region taping. The center of force and mean peak gluteal pressure were measured using a TekScan system. [Results] With extensive taping, the AP center of force progressively decreased after 10,20, and $30 \mathrm{~min}$, while the ML center of force differed only slightly by time point. With lengthwise taping, the ML and AP center of force decreased after $20 \mathrm{~min}$. [Conclusion] These findings suggest that lengthwise taping is a useful method for patients having lumbar rotation with flexion syndrome to prevent slumped sitting posture and excessive pressure on the gluteal muscles.

Key words: Lumber rotation with flexion syndrome, Postural taping, Prolonged sitting
\end{abstract}

(This article was submitted Jan. 24, 2018, and was accepted Apr. 13, 2018)

\section{INTRODUCTION}

Recent studies have identified the importance of categorizing individuals with low back pain (LBP) into homogeneous subgroups to design more effective interventional strategies ${ }^{1}$. According to this paradigm, sustained prolonged sitting may lead to easier rotation in flexion direction-specific lumbo-pelvic movement patterns during daily activity because the sitting position promotes a greater degree of rotation compared to the upright position, for example in individuals who sit in a slumped position followed by rotation to reach a computer or files ${ }^{1)}$. The self-conscious lumbo-pelvic control method has been recommended as a safe approach to correct poor posture ${ }^{2}$. Comerford and Mottram ${ }^{2}$ suggested thatan individual should assume various positionswhile lumbo-pelvic motion is monitored by self-palpation, or taping of the L2, L5, and S2 spinous processes for effective flexion movement control. In the current study, we investigated the effectiveness of postural taping for preventing asymmetry and a slumped sitting posture in a patient having lumbar spine rotation with flexion syndrome during a long period ofsitting while working at a computer.

\section{SUBJECT AND METHODS}

The subject was a 22-year-old woman who had developed transient LBP in the lumbo-pelvic region (visual analog pain score=3) during a 2-hour sitting period. The subject had no other diseases such as metabolic, neuromuscular, upper or lower extremity disorders or history of spinal surgery. The study adhered to the principles of the Declaration of Helsinki and its purpose and methods were explained to the participant before the study commenced; she also provided informed consent.

*Corresponding author. Won-Gyu Yoo (E-mail: won7y@inje.ac.kr)

(C2018 The Society of Physical Therapy Science. Published by IPEC Inc.

(c) $(-)$ This is an open-access article distributed under the terms of the Creative Commons Attribution Non-Commercial No Derivatives

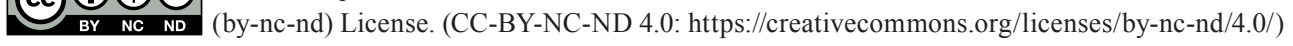


The subject complained of transient LBP during prolonged sitting twice per month for 3 months, but had received no specific treatment for this condition. One examiner evaluated her active movement using a protocol designed to categorize individuals with LBP into homogenous subgroups as described by Sahrmann ${ }^{1)}$. Based on her movement system impairment, her condition was classified as rotation with flexion syndrome. The subject was asked to sit in a chair during a 30-minute period of computer work under three conditions: (1) without taping, (2) with extensive taping ${ }^{3)}$, and (3) with lengthwise taping ${ }^{2)}$. To restrict excessive lumbar rotation with flexion during the computer work, we used non-elastic tape (Endura Sports Tape; Endura-Tape, Sydney, Australia) on lumbo-pelvic region for the postural taping. To induce normal curve of lumbar spine, the participant held a posture with slight lumbar extension in the prone position. An examiner applied the extensive taping in the following manner. Hypoallergenic tape (Endura Fix Tape, Endura-Tape Pty. Ltd.) was attached under the non-elastic tape to minimize effects on the skin. Tapes were attached transversely to the back across the 1st lumbar spine and bilateral posterior superior iliac spine for superior and inferior anchors, respectively and then one longitudinal tape connected the superior anchor to the inferior anchor through the lumbar spinous processes, and the others were connected between the ends of the superior anchor and the ends of the inferior anchor. One longitudinal tape attached the spinous process of 1st lumbar spine and midpoint of posterior superior iliac spine. The subject sat with her hips and knees flexed at $90^{\circ}$; a height-adjustable table was used to set the initial sitting posture. The center of force distribution and mean peak pressure of the left and right gluteal portions were recorded at 10, 20, and 30 minutes using the CONFORMat system (TekScan, Boston, MA, USA).

\title{
RESULTS
}

Without taping, the medio-lateral (ML) center of force progressively increased after 10, 20, and 30 minutes $(1,216.0$, $1,577.5$, and $2,009.8 \mathrm{~cm} / \mathrm{s}^{2}$, respectively), and to a greater degree than did the antero-posterior (AP) center of force $(1,142.3$, $1,087.17$, and $1,261.7 \mathrm{~cm} / \mathrm{s}^{2}$, respectively). With extensive taping, the AP center of force progressively decreased after 10 , 20 , and 30 minutes $\left(1,397.6,1,085.4\right.$, and $977.5 \mathrm{~cm} / \mathrm{s}^{2}$, respectively), and that of the ML was similar $(1,397.6,1,343.1$, and $1,232.3 \mathrm{~cm} / \mathrm{s}^{2}$, respectively). With lengthwise taping, the center of force for both ML $\left(1176.3,1136.05\right.$, and $830.3 \mathrm{~cm} / \mathrm{s}^{2}$, respectively) and AP $\left(1263.4,1204.19\right.$, and $893.1 \mathrm{~cm} / \mathrm{s}^{2}$, respectively) experienced the greatest decrease after $20 \mathrm{~min}$.

\section{DISCUSSION}

Our findings indicate that lengthwise lumbo-pelvic region taping is a more effective method for reducing the center of force compared to extensive taping and no taping during prolonged sitting, although extensive taping was more effective than no taping in reducing AP and ML movement. Greig et al. ${ }^{4)}$ reported significantly reduced thoracic kyphosis immediately after the application of non-elastic tape during postural tape in a standing position. In the dynamic activities such as walking or transfer, the extensive taping method was effective that maximal lumbar flexion angle and the erector spinae muscle activities would be decreased ${ }^{3}$. However, in this study showed that in static posture, excessive taping shows less effectiveness. This result also indicated that the taping method may be applied differently depending on the type of activities. Furthermore, because we applied the tape with the normal curve maintained, if the participants flexes excessively lumbar spine during task, participants may feel the skin pulling. It may provide an opportunity to do correct the wrong posture yourself. For this reason, we consider lengthwise taping a more valid approach than extensive taping, because lengthwise taping provides proper mechanical support to prevent excessive trunk flexion and rotation and allows movement for repositioning.

\author{
Funding \\ None. \\ Conflict of interest \\ None.
}

\section{REFERENCES}

1) Sahrmann S: Diagnosis and treatment of movement impairment syndromes. New York: Mosby, 2002.

2) Comerford M, Mottram S: Kinetic control: the management of uncontrolled movement. Australia: Churchill Livingstone, 2012.

3) Kang MH, Choi SH, Oh JS: Postural taping applied to the low back influences kinematics and EMG activity during patient transfer in physical therapists with chronic low back pain. J Electromyogr Kinesiol, 2013, 23: 787-793. [Medline] [CrossRef]

4) Greig AM, Bennell KL, Briggs AM, et al.: Postural taping decreases thoracic kyphosis but does not influence trunk muscle electromyographic activity or balance in women with osteoporosis. Man Ther, 2008, 13: 249-257. [Medline] [CrossRef] 